Ann. Génét. Sél. anim., I978, 10 (4), 557-568.

\title{
Estimation, à l'aide d'une lignée-témoin, del'évolution génétique des caractères d'engraissement et de carcasse du Porc Large White en France, de 1965 à 1973
}

\author{
Y. HOUIX, P. DANDO* et P. SELLIER \\ avec la collaboration technique de A. DERRIEN et D. TASTU \\ Station de Génétique quantitative et appliquée \\ Centre national de Recherches zootechniques, I.N.R.A., \\ 78350 Jouy-en-Josas \\ *Domaine de Galle, Avord, I88oo Baugy
}

\begin{abstract}
Résumé
En l'absence d'un véritable troupeau-témoin porcin en France, il a paru possible d'utiliser comme tel, vis-à-vis des caractères de croissance et de carcasse, une lignée expérimentale de l'I.N.R.A., entretenue au domaine de Galle (Avord, Cher). Cette lignée de porcs Large White a été constituée en 1965 en vue d'une expérience de sélection sur la prolificité et n'a été soumise depuis lors à aucune sélection directe sur les caractères de croissance et de carcasse, ni à aucune sélection indirecte si on admet que ces caractères sont génétiquement indépendants de la taille de portée. A fin de mesurer l'évolution génétique des caractères de production de 1965 à 1973 dans la base de sélection du Large White en France, roo femelles issues du troupeau de Galle ont donc été comparées, dans les stations de contrôle de descendance, à I 6 o femelles contemporaines provenant de plus de 30 élevages de sélection de la race Lavge White. Les résultats traduisent une évolution génétique significative et favorable de cette race, à la fois pour le gain moyen quotidien $(+\mathbf{I} \mathbf{g} /$ an) et l'indice de consommation (- $-0,05$ point /an) et pour la plupart des critères de composition corporelle $(-0,8 \mathrm{~mm} /$ an pour l'épaisseur de lard dorsal, $+0,5$ point/an pour le pourcentage estimé de muscle). Le progrès génétique annuel peut être chiffré à environ $7,5 \mathrm{~F}$ pour la marge brute par porc engraissé de 25 à $100 \mathrm{~kg}$. Dans le même temps, une diminution significative du $\mathrm{pH}$ ultime du muscle a été trouvée mais les autres critères indicateurs de la qualité technologique de la viande n'ont pas évolué de façon significative. Les hypothèses sur lesquelles repose la validité de ces estimations d'évolution gétiétique sont discutées.
\end{abstract}

\section{Introduction}

L'importance des moyens financiers engagés par la collectivité pour l'amélioration génétique des animaux domestiques impose de s'assurer a posteriori de l'efficacité des plans de sélection mis en place. Les différentes méthodes de 
mesure du progrès génétique dans les populations animales, passées en revue et discutées par HILI. (I972a), ColıEAU (I975a et $b$ ) et SMiTh (I976, I977), reposent toutes sur la comparaison d'individus de générations (ou cohortes) différentes placés dans le même milieu. Des méthodes d'estimation des évolutions génétiques dans le temps sans population-témoin, en particulier celle proposée par SMITH ( I962), ont été utilisées dans le cas du Porc, comme par exemple dans les études de Smith (I963), Cox et Smith (I968), Naveau (I97I) et Olirivier (I974, I977). Une autre méthode classique de mesure du progrès génétique dans les populations animales consiste à comparer celles-ci à un troupeau-témoin maintenu sans sélection. Dans l'espèce porcine, de tels troupeaux ont été constitués dans les années soixante en Grande-Bretagne (Cook et al., I97I).

A l'heure actuelle en France, il n'existe pas de troupeau-témoin porcin au sens strict mais il a paru possible d'utiliser comme tel un troupeau expérimental de 1'I.N.R.A., créé en r 965 dans un tout autre but et entretenu au domaine de Galle, à Avord (Cher). Depuis sa création, cette lignée de porcs Large White a été sélectionnée sur la taille de portée à la naissance (OLLIVIER, I973) et n'a été soumise à aucune sélection sur les caractères de croissance et de carcasse. Dans la mesure où l'échantillon des animaux achetés en I 965 (I20 truies et ro verrats provenant d'une dizaine d'élevages, pour la plupart inscrits au Livre Généalogique et pratiquant de façon régulière le contrôle de descendance des verrats) était représentatif de la population Large White de l'époque et où l'on fait l'hypothèse qu'il y a indépendance génétique entre la prolificité et les caractères de production, comme le montrent les résultats obtenus dans cette race par LEGAULT (I97I), le troupeau du domaine de Galle peut être considéré comme une lignée-témoin pour les caractères d'engraissement et de carcasse et utilisé pour la mesure de l'évolution génétique de ces caractères depuis I 965 dans la base de sélection du porc Large White en France.

A cette fin, des jeunes femelles provenant de la lignée de Galle (" $L W$ Galle ") ont été envoyées en I973-I974 dans les stations de contrôle de descendance et comparées à des jeunes femelles Large White contemporaines provenant d'élevages de sélection adhérents de 1'U.P.R.A. (" $L W U P R A$ »). Nous présentons ici le bilan final de cette comparaison dont les résultats préliminaires avaient été publiés par Houix et al. (I975).

\section{Matériel et méthodes}

\section{I. - Matériel animal}

Les données analysées portent sur roo femelles $L W$ Galle et I6o femelles $L W U P R A$ réparties dans 8 bandes de contrôle. Les roo femelles $L W$ Galle sont issues de 8 pères différents (nés en I972) et proviennent de 3 séries de mise bas. La plupart des mises bas de la $2^{\mathrm{e}}$ série et les mises bas de la $3^{\mathrm{e}}$ série ont eu lieu au Lycée agricole de Clermont-Ferrand-Marmilhat (Puy-de-Dôme) où un échantillon de verrats et de truies de la lignée de Galle avait été transféré en r974. Les I6o femelles $L W$ UPRA contemporaines sont filles de 45 pères différents et proviennent de plus de 30 élevages de sélection de la race. 


\section{2. - Caractères mesurés}

Ces animaux ont été soumis aux modalités de contrôle habituelles des stations de contrôle de descendance (Molenat et al., I974). Les jeunes femelles entrent en station à un poids compris entre 22 et $28 \mathrm{~kg}$, sur une période de 2 semaines pour une bande donnée. Elles sont logées par lot de deux pleines-sœurs etle contrôle de croissance et de consommation d'aliment débute quand le poids moyen du lot atteint $35 \mathrm{~kg}$. L'aliment est distribué à volonté. Les deux sœurs d'un lot sont abattues le même jour quand leur poids vif moyen atteint roo kg. L'indice de consommation et la consommation moyenne journalière d'aliment sont calculés par lot.

Le lendemain de l'abattage, la carcasse (sans tête) est pesée, la longueur de la carcasse (atlas-pubis) et les épaisseurs de lard dorsal au niveau de la dernière vertèbre lombaire (rein), de la dernière vertèbre dorsale (dos) et de la dernière vertèbre cervicale (cou) sont mesurées et une demi-carcasse est soumise à la découpe parisienne normalisée (OlLIVIER, I970). Une section transversale de l'ensemble longe-bardière est réalisée entre $1 \mathrm{a} I 3^{\mathrm{e}}$ et la $\mathrm{I} 4^{\mathrm{e}}$ côte : les surfaces de la noix de côtelette et du gras sus-jacent sont mesurées par planimétrie sur un calque de la coupe.

Par ailleurs, une note subjective de qualité de viande est donnée et trois critères indicateurs de la qualité technologique de la viande sont mesurés 24 heures post mortem:

- le $\mathrm{pH}$ du muscle Adducteur (Adductor femoris),

- la réflectance du muscle Fessier superficiel (Gluteus superficialis), déterminée à l'aide du réflectomètre Manuflex de Vergé-Nicou,

- le pouvoir de rétention d'eau du muscle Long vaste (Biceps femoris), apprécié par le temps d'imbibition d'un papier $\mathrm{pH}$ appliqué sur la surface du muscle fraîchement coupé, la durée de l'observation étant limitée à $3 \mathrm{mn}$.

\section{3. - Analyse statistique}

La différence entre $L W U P R A$ et $L W$ Galle a été estimée pour 24 variables par la méthode des moindres carrés, appliquée à un modèle à effets fixés (HARVEy, I975). Les effets du modèle utilisé sont :

- le type génétique ( 2 niveaux), la bande de contrôle ( 8 niveaux), 1'interaction type génétique $\times$ bande et la régression sur le poids de début de contrôle pour le gain moyen quotidien,

- les mêmes effets et en plus la régression sur le poids moyen du lot en fin de contrôle pour l'indice de consommation et la consommation journalière d'alimenit,

- le type génétique, la bande de contrôle, 1'interaction type génétique $x$ bande et la régression linéaire sur le poids vif d'abattage pour les variables de composition corporelle, y compris le rapport longe/bardière et les pourcentages de muscle et de gras dans la carcasse avec tête, estimés à l'aide des poids de morceaux de la découpe selon les fonctions établies par HAMELIN (I975, communication personnelle) et données par SELLIER (I977),

- le type génétique, la station de contrôle (4 niveaux), la date d'abattage ( 52 niveaux) et la régression linéaire sur le poids vif d'abattage pour les 4 variables de qualité de viande. 
Pour le calcul de l'erreur-standard de la différence $d=L W U P R A-L W$ Galle, il a été tenu compte à la fois de la variance d'échantillonnage $\sigma_{\mathrm{E}}^{2}$ de l'estimée de cette différence et de la variance $\sigma^{2}$ due à la dérive génétique dans le troupeautémoin (HILL, I972a). Le terme $\sigma^{2}$ a été calculé à l'aide des éléments de l'inverse de la matrice des moindres carrés. La variance de dérive génétique $\sigma^{2}{ }_{D}$ a été prise égale à $2 F h^{2} s^{2}$, où :

$F$ est le coefficient de consanguinité moyen du troupeau-témoin, calculé à partir des pedigrees des porcs $L W$ Galle contrôlés et trouvé égal à 0,063 ;

$h^{2}$ est l'héritabilité du caractère : les valeurs de $h^{2}$ trouvées par OLLIVIER (I970) chez le Large White français ont été utilisées;

$s^{2}$ est la variance phénotypique du caractère, estimée intra-type génétique et intra-bande de contrôle.

Cette expression de $\sigma^{2}$ ne vaut en toute rigueur que pour des caractères dont toute la variance génétique est additive mais, d'après HILL (I972b), elle reste une bonne approximation pour les caractères affectés par des effets de dominance, du moins tant que la valeur de $F$ est, comme c'est le cas ici, relativement faible.

L'hypothèse $d=0$ a été testée à l'aide du $t$ de Student.

\section{4. - Évaluation économique des gains génétiques}

Afin de préciser, en termes d'économie de la production porcine, l'importance des évolutions génétiques observées, nous avons comparé le $L W U P R A$ et le $L W$ Galle sur la base d'une fonction économique qui vise à estimer la marge brute $M$ par porc engraissé de 25 à roo kg; $M$ est égal à la différence entre la valeur commerciale de la carcasse $(R)$ et le coût d'engraissement $(C)$, avec :

$$
R=306,3+\mathrm{I} 4,88 J+\mathrm{I}_{5,96} L-\mathrm{I} 2,60 B
$$

où $J, L$ et $B$ sont respectivement les poids (en $\mathrm{kg}$ ) du jambon, de la longe et de la bardière dans une demi-carcasse,

$$
C=A+0,24 D
$$

où $A$ est la consommation totale d'aliment (en $\mathrm{kg}$ ) et $D$ est la durée d'engraissement (en jours).

Pour le calcul de $C$, nous disposions des valeurs de $A$ et $D$ relatives à la période de contrôle 35 -roo $\mathrm{kg}$ et pour passer à la période 25 -Ioo $\mathrm{kg}$, qui a plus de signification dans les conditions pratiques de production, nous avons supposé que les différences entre types génétiques pour le gain moyen quotidien et l'indice de consommation étaient les mêmes pour les deux périodes. Les paramètres économiques utilisés correspondent aux conditions économiques de la production porcine dans les années r975-1976.

\section{Résultats}

Pour chacune des 24 variables étudiées, nous rapportons dans le tableau I la différence entre $L W U P R A$ et $L W$ Galle, son degré de signification statistique et l'estimation de l'évolution génétique annuelle. Ces chiffres indiquent un avan- 
TABLEAU I

Estimées de la différence $\mathrm{d}=\mathrm{LW}$ UPRA - $\mathrm{LW}$ Galle pour les caractère étudiés Estimates of the difference between $\mathrm{LW} \mathrm{UPRA}$ and $\mathrm{LW}$ Galle for the traits studied

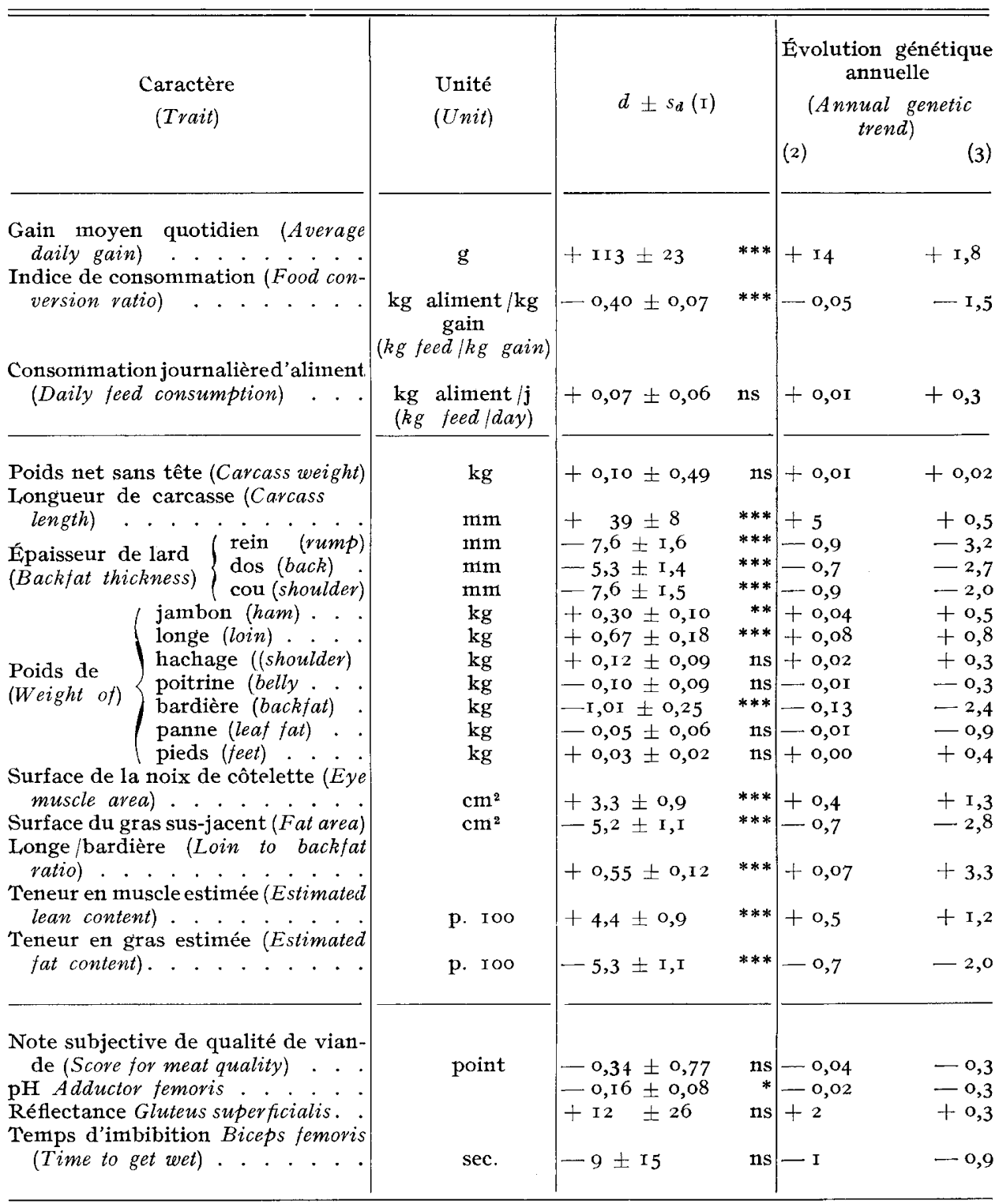

(1) $s_{d}=$ erreur-standard de $d$ (standard error of d). Signification statistique (Statistical significance): ns : $\mathrm{P}>0, \mathrm{IO} \quad * *: \mathrm{P}<\mathrm{O}, \mathrm{Or}$

$*: \mathrm{P}<0,05 \quad * * *: \mathrm{P}<0,00 r$.

(2) En unité du caractère (In unit of the trait).

( ${ }^{3}$ En p. roo de la moyenne des moindres carrés (As a percentage of the least-squares mean). 
tage très hautement significatif $(\mathrm{P}<\mathrm{o}$,oor $)$ des porcs $L W U P R A$ pour le gain moyen quotidien $(832$ contre $7 \mathrm{I} 9 \mathrm{~g} / \mathrm{j})$, l'indice de consommation $(3,09$ contre 3,49 ) et la plupart des critères de composition corporelle, comme par exemple l'épaisseur moyenne du lard dorsal (30,I contre 37,0 $\mathrm{mm}$ ) et la teneur estimée en muscle de la carcasse avec tête $(49,2$ contre 44,8 p. Ioo). La consommation journalière d'aliment est un peu plus forte chez le $L W U P R A(2,57 \mathrm{~kg} / \mathrm{j})$ que chez le $L W$ Galle $(2,50 \mathrm{~kg} / \mathrm{j})$ mais la différence n'est pas significative. Le rendement en carcasse est le même chez les deux types de porcs. La réduction notable de l'adiposité de la carcasse se manifeste au niveau du dépôt de gras dorsal mais par contre les poids de panne et de poitrine ne sont pas significativement plus faibles chez le $L W U P R A$. L'accroissement du poids des morceaux nobles est particulièrement net pour la longe dont l'augmentation de poids est à relier à la fois à une plus grande surface de noix de côtelette et à une longueur accrue de la carcasse. Un dernier point à souligner concerne la qualité technologique de la viande qui tend à évoluer de façon défavorable dans la population Large White française, si l'on en juge par le $\mathrm{pH}$ ultime du muscle Adducteur significativement plus faible chez le $L W U P R A$. Toutefois les deux types de porcs ne diffèrent pas de façon significative pour les autres critères de qualité de viande.

\section{Discussion}

Ces résultats traduisent une évolution génétique favorable des élevages de sélection du porc Large White en France entre I965 et I973, à la fois pour les performances de croissance et la composition tissulaire de la carcasse mais la validité de cette estimation de progrès génétique repose sur un certain nombre d'hypothèses qui méritent d'être discutées.

En premier lieu, il est supposé implicitement que l'échantillon des animaux fondateurs du troupeau de Galle est représentatif du niveau génétique moyen des élevages inscrits au Livre Généalogique du Large White en I965. Les éléments d'information font défaut pour vérifier cette hypothèse mais il est permis de penser que la variété d'origine des animaux ayant servi à la constitution du troupeau rend peu probable un biais initial d'échantillonnage.

Une autre source possible de biais est l'existence d'une évolution génétique orientée des caractères étudiés dans le troupeau de Galle. En dehors des effets de la sélection naturelle vraisemblablement très faibles pour des caractères peu liés à la fertilité et à la viabilité, deux causes principales peuvent a priori contribuer, dans le cas présent, à ce type d'évolution génétique : la dépression due à la consanguinité d'une part, les réponses corrélatives à la sélection sur la taille de portée d'autre part.

La faible valeur du coefficient de consanguinité moyen du troupeau de Galle au moment de la comparaison (moins de $7 \mathrm{p}$. Ioo pour les animaux contrôlés) limite l'incidence du premier facteur. Il ne jouerait de toute façon que pour le gain moyen quotidien et l'indice de consommation, les caractères de carcasse n'étant pas affectés de façon sensible par la consanguinité.

Bien qu'en principe, une lignée-témoin ne doive être soumise à aucune sélection, une lignée sélectionnée sur un caractère $X$ (taille de portée) peut servir de témoin vis-à-vis d'une population sélectionnée sur un ensemble de caractères $Y$ (performances d'engraissement et de carcasse), si $\mathrm{X}$ et $\mathrm{Y}$ ne sont pas liés génétiquement. Cette hypothèse d'indépendance génétique est évidemment une condi- 
tion essentielle de validité de notre estimation. Au vu des très faibles corrélations génétiques trouvées entre la taille de portée et les caractères de production, chez le Large White français par LEGAULT (I97I) et chez les Large White et Landrace britanniques par MoRRIS (I975), cette hypothèse nous paraît tout à fait acceptable. Elle est également étayée par l'absence de réponse corrélative significative sur la prolificité dans plusieurs expériences de sélection sur des caractères de production, tels que la vitesse de croissance (GARNETT et RAHNEFELD, I976) et 1'épaisseur de lard dorsal (GRAy et al., I965; HETZER et MILLER, I970), ou sur un indice combinant plusieurs caractères de production (CHADWICK et al., I976; WEBB et KING, I976). Indiquons également que dans l'expérience de sélection sur le taux d'ovulation réalisée à l'Université du Nebraska, aucune évolution génétique de la vitesse de croissance et de la composition corporelle n'a été enregistrée (NEWTON et al., I977; ENGLAND et al., I977). Une des seules liaisons génétiques notables observées par LEGAULT (I97I) concerne la taille de portée à la naissance et la longueur de carcasse $\left(r_{g}=-0,33\right)$ : dans le cas présent, cette liaison a pu entraîner une réduction de la longueur dans le troupeau de Galle et donc contribuer à une surestimation de 1'accroissement de longueur des porcs Large White entre I965 et 1973. Par ailleurs, une corrélation génétique fortement négative $(-0,5 \mathrm{a}-0,6)$ entre la taille de portée et le rendement en carcasse a été trouvée dans les deux races étudiées par MorRIs (I975) : sur cette base, il se pourrait donc que le troupeau de Galle ait connu une diminution du rendement en carcasse et que ce soit également le cas pour la population $L W U P R A$ puisqu'aucune différence n'a été trouvée de ce point de vue dans la présente comparaison. Qu'il s'agisse de la longueur ou du rendement, leur diminution dans le troupeau de Galle, comme conséquence de la sélection en faveur de la prolificité, ne doit être considérée que comme une possibilité; leur liaison génétique avec la taille de portée n'est pas établie de façon certaine, comme le montrent plusieurs autres études citées par LEGAULT (I97I).

Sur un tout autre plan, il est bon d'avoir à l'esprit que la présente comparaison entre $L W U P R A$ et $L W$ Galle n'a porté que sur des femielles nourries à volonté. Des interactions entre le type génétique d'une part, le sexe (femelle ou mâle castré) ou le niveau d'alimentation (à volonté ou restriction) d'autre part ont parfois été mises en évidence chez le porc en croissance dans des expériences de comparaison entre types génétiques. En particulier, dans plusieurs études portant sur les lignées " grasse ", " contrôle " et " maigre " de l'expérience de sélection sur l'épaisseur de lard dorsal réalisée à la station de Beltsville, une interaction sexe $\times$ lignée a été mise en évidence pour la plupart des caractères de composition corporelle d'animaux nourris à volonté, la différence entre lignées étant plus forte chez les femelles que chez les mâles castrés (HETZER et MILLER, I973; BERESKIN et DAVEY, I976; DAVEy et BERESKIN, I978). Il est vrai que ce résultat a été trouvé sur des types génétiques présentant de très grandes différences d'adiposité de la carcasse mais le fait de comparer seulement des femelles a pu conduire à une certaine surestimation de l'avantage de composition corporelle du type génétique le plus maigre, c'est-à-dire du $L W U P R A$.

Ces diverses réserves étant faites, le progrès génétique réalisé chez le Large White français sur la période de 8 ans considérée peut, en termes économiques, être chiffré à environ $6 \mathrm{I} F$ pour la marge brute par porc engraissé entre 25 et Ioo $\mathrm{kg}$, ce qui représente un progrès génétique annuel de 1'ordre de $7,5 \mathrm{~F}$. Cet accroissement de la marge brute unitaire provient de façon à peu près égale, en valeur absolue, de la réduction du coût d'engraissement $(-4 \mathrm{~F} / \mathrm{an})$ et de 1'augmentation de la valeur commerciale de la carcasse $(+3,5 \mathrm{~F} / \mathrm{an})$. Exprimée en pour- 
centage de la moyenne, 1'évolution génétique annuelle est de 1'ordre de - I,6 p. Ioo pour le coût d'engraissement et de $+0,7$ p. Ioo pour la valeur commerciale de la carcasse.

Sur cette même population Large White française, OLLIVIER (I974) a précédemment estimé, sur la base de la comparaison intra-année de la descendance de verrats d'âges différents, la part génétique des changements survenus entre I953 et I 966 pour les performances d'engraissement et de carcasse. Pendant cette période, une réduction importante du coût d'engraissement (à raison de $2,2 \mathrm{p}$. Ioo par an pour la part d'origine génétique) avait été enregistrée mais, par contre, la composition corporelle avait évolué génétiquement de façon défavorable $(-0,6 \mathrm{p}$. roo par an pour le prix de carcasse). La comparaison des estimations relatives aux périodes I 953 - I966 et I965-I973 semble donc indiquer que le progrès génétique annuel sur les composantes du coût d'engraissement a été du même ordre de grandeur dans les deux périodes et que, depuis 1965 , à cette réduction du coût d'engraissement s'est ajoutée une amélioration génétique notable de la qualité de carcasse. Au total, d'une période à l'autre, le progrès génétique annuel en race Large White pour la marge brute unitaire a plus que doublé $(7,5 \mathrm{~F}$ contre $3 \mathrm{~F})$. Parmi les causes possibles de cet accroissement d'efficacité de la sélection, le développement rapide des stations de contrôle individuel des verrats à partir de I966 (OLLIVIER et al., I978) mérite une mention toute particulière. Dans la période antérieure, les éleveurs-sélectionneurs n'avaient à leur disposition que le contrôle de descendance des verrats et, comme 1'ont constaté CzAJEWSKA et OLIIVIER (I969), cet " outil de sélection " était mal utilisé : le nombre de verrats contrôlés annuellement était très faible et le taux d'élimination sur les résultats de l'épreuve de descendance était proche de zéro. Le contrôle individuel des verrats porte sur un nombre beaucoup plus grand d'animaux et conduit à une sélection effective. Certes, le fonctionnement des stations de contrôle individuel n'a atteint sa "vitesse de croisière " que vers la fin de la période considérée ici mais les effets bénéfiques de cette nouvelle méthode de sélection sur le progrès génétique s'étaient déjà probablement fait sentir, comme le suggère le premier bilan établi par MoLENAT et Naveau (I970).

I1 est intéressant de rapprocher les évolutions génétiques annuelles observées ici des réponses obtenues dans l'expérience de sélection sur indice de contrôle individuel de la population Large White du Poitou (Oldivier, I977). Cette expérience, commencée en I 965 et réalisée dans le cadre du centre d'insémination artificielle de Rouillé, peut être considérée d'une certaine manière comme un " modèle réduit " du plan national de sélection sur les caractères de production. Les évolutions génétiques annuelles chez le $L W U P R A$ et chez le $L W$ Poitou sont du même ordre de grandeur pour les performances de croissance mais les évolutions génétiques favorables de 1'épaisseur de lard et du pourcentage estimé de muscle sont sensiblement plus fortes chez le $L W U P R A$ que chez le $L W$ Poitou. Il vaut d'ailleurs d'être noté que la comparaison en croisement de verrats représentant le niveau génétique de ces deux populations en I973-74 (SELLIER, I977) a justement mis en évidence une légère " avance " du $L W U P R A$ pour la composition tissulaire de la carcasse. D'autre part, dans un cas comme dans l'autre, l'évolution génétique de l'appétit n'est pas significative mais la tendance observée va dans le sens d'une augmentation de la consommation journalière d'aliment. Enfin l'augmentation d'origine génétique du poids de tête relevée chez le $L W$ Poitou et associée à la moindre maturité physiologique des animaux au poids vif de Ioo $\mathrm{kg} \mathrm{n}$ 'a pas pu être confirmée par la présente étude, le poids de tête n'étant pas mesuré dans les stations de contrôle de descendance. 
TABLEAU 2

Évolutions génétiques annuelles des caractères de production dans les élevages de sélection des Large White et Landrace britanniques, estimées à l'aide de troupeaux-témoins

Annual genetic trends of production traits in British Large White and Landrace selection herds, estimated with control herds

\begin{tabular}{|c|c|c|c|c|}
\hline Référence (Source) & Cook et al.(197I) & $\begin{array}{c}\text { MEAT } \\
\text { and LrVESTOCK } \\
\text { CoMMISSION }\end{array}$ & JONES & I978) \\
\hline Race (Breed) & Large White $(a)$ & Large White $(a)$ & Large White $(b)$ & Landrace $(c)$ \\
\hline $\begin{array}{l}\text { (Trait) } \\
\text { (Periode }) \\
\end{array}$ & $1967-\mathrm{I} 97 \mathrm{I}$ & I 969-I 974 & 1972-1977 & I 972-I977 \\
\hline $\begin{array}{l}\text { Gain moyen quotidien (g) } \\
\text { (Average daily gain) }\end{array}$ & $+\mathbf{1} 8^{*}$ & $+\mathbf{r} \mathrm{ns}$ & $+8^{* *}$ & $+9^{* *}$ \\
\hline $\begin{array}{l}\text { Indice de consommation } \\
\text { (Food conversion ratio) }\end{array}$ & $-0,055^{* *}$ & $-0,020^{* *}$ & $-0,03 I^{* *}$ & $-0,08^{* *}$ \\
\hline $\begin{array}{l}\text { Pourcentage de maigre dans } \\
\text { le " rein arrière " . } \\
\text { (Lean percent in vump back) } \\
\text { Surface de la noix de côte- }\end{array}$ & $+1,60 * *$ & $+0,45 \mathrm{~ns}$ & $+0,65^{* *}$ & $+\mathrm{I}, \mathrm{I} 4^{* *}$ \\
\hline $\begin{array}{c}\text { lette }\left(\mathrm{cm}^{2}\right) \\
(\text { Eye muscle area })\end{array}$ & $+0,63^{* *}$ & $+0,4^{* * *}$ & $+0,44^{* *}$ & $+0,25^{* *}$ \\
\hline $\begin{array}{l}\text { Rendement (p. 100) } \\
\text { (Killing out percentage) } \\
\text { Épaisseur de lard au point } \mathrm{C}\end{array}$ & $-0,29^{*}$ & $+0,12^{*}$ & $+0,12 *$ & $+0,2 I^{* *}$ \\
\hline 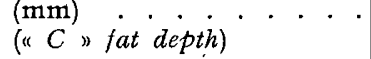 & & $-0,45^{* *}$ & $-0,42^{* *}$ & $-0,75^{* *}$ \\
\hline $\begin{array}{l}\text { Valeur économique globale } \\
\text { par porc (pence) . } \\
\text { (Overall economic merit per } \\
\text { pig) }\end{array}$ & +44 & & +73 & +106 \\
\hline
\end{tabular}

(a) Résultats combinés de 2 troupeaux-témoins (Newcastle et Bangor).

(b) Un troupeau-témoin (Newcastle).

(c) Un troupeau-témoin (Wye).

A notre connaissance, la Grande-Bretagne est le seul pays où l'évolution génétique de populations porcines " nationales " (Large White et Landrace) est mesurée de façon régulière depuis la fin des années 60 à l'aide de troupeauxtémoins entretenus dans ce but. Les résultats de cette mesure de progrès génétique présentent un intérêt tout particulier et nous avons rapporté dans le tableau 2 les évolutions génétiques de quelques caractères sur différentes périodes dans les deux races britanniques. Confronter les chiffres du tableau 2 et nos propres estimations sur le Large White français n'est pas sans poser des problèmes car les caractères mesurés et les conditions de leur mesure diffèrent selon le pays mais il semble que les évolutions génétiques sont, d'une manière générale, du même ordre de grandeur dans les deux pays. Il est important de rappeler que dans un cas comme dans l'autre, il s'agit d'évolutions génétiques dans les élevages de sélection : il resterait à préciser dans quelle mesure ce progrès génétique diffuse dans l'ensemble de la population porcine. 
Le dernier point à souligner concerne l'évolution génétique des critères de qualité de viande. Le problème des effets indirects d'une sélection visant à améliorer la vitesse de croissance et à réduire 1'adiposité de la carcasse est encore très controversé. Dans le cas présent, la seule évolution génétique significative ( $-0,02$ point/an pour le $\mathrm{pH}$ ultime du muscle Adducteur) est défavorable en termes de qualité technologique de la viande. Ce résultat doit, à notre avis, être considéré avec prudence et demanderait confirmation, dans la mesure où la couleur et le pouvoir de rétention d'eau de la viande, pourtant étroitement liés au $\mathrm{pH}$, n’ont pas évolué de façon significative. Les résultats disponibles dans la littérature dans ce domaine sont rares et peu concordants. Après dix générations de sélection sur un indice combinant gain moyen quotidien et épaisseur de lard dorsal, dans une expérience conduite en Norvège (VANGEN, 1977), la couleur du muscle Long dorsal est devenue plus pâle dans la lignée " haute " $(\mathrm{P}<0, \mathrm{or})$ et aussi dans la lignée " basse " mais de façon non significative. Sur le même plan, les résultats de l'expérience de sélection d'OLLIVIER (I977) sont assez disparates mais, dans l'ensemble, les faibles vitesses d'évolution observées incitent à penser que l'amélioration génétique de la quantité de viande dans la carcasse n'a pas entraîné une dégradation de sa qualité. C'est à cette même conclusion qu'arrivent CHADwick et al. (1976) dans une autre expérience de sélection sur indice.

Rę̧u pour publication en février 1979.

\title{
Remerciements
}

Une aide matérielle a été accordée en 1974 par le Ministère de 1'Agriculture au Lycée agricole de Marmilhat pour la réalisation de cette expérimentation. Nous remercions M. ECHEGUT, directeur de ce Lycée agricole et MM. BLANCHON et RAFFIER de leur collaboration. Nous remercions également pour leurs utiles observations les lecteurs du manuscrit J. J. CoLLEAU, M. MOLENAT et L. Olitivier (I.N.R.A., Jouy-en-Josas). Nous tenons enfin à remercier D. E. STEANE (Meat and Livestock Commission) de nous avoir communiqué le plus récent rapport interne du M.L.C. sur les troupeaux-témoins britanniques.

\section{Summary}

\author{
Estimation, using a control herd, of genetic change of growth \\ and carcass traits in the French Large White breed from I965 to I973
}

No pig control herd as such has so for been established in France. However, an I.N.R.A. experimental line, kept at the " domaine de Galle" (Avord, Cher), could be used as a control with respect to production traits. This line of Large White pigs, established in 1965 for a selection experiment on litter size at birth, has not been since submitted to any direct selection on growth and carcass traits, nor to any indirect selection of these traits which are assumed to be genetically independent of litter size. In order to estimate the genetic trends of production traits in the French Large White population on a 8-year period (1965-1978), roo female pigs from the Galle herd ( $L W$ Galle) have been compared, in progeny-testing stations, with I6o contemporary female pigs from more than 30 selection herds of the Large White breed ( $L W U P R A)$. Results show significant and favourable genetic trends in this breed, both for rate and economy of gain ( $+14 \mathrm{~g}$ per year in average daily gain,- 0.05 point per year in food conversion ratio) and for most carcass composition traits $(-0.8 \mathrm{~mm}$ per year in backfat thickness, +0.5 point fer year in estimated percentage of lean). The estimated annual genetic gain is around $7.5 \mathrm{~F}$ in profit per pig fattened from 25 to roo $\mathrm{kg}$ liveweight. In the same time, a significant decrease of ultimate $\mathrm{pH}$ of muscle has been observed but the other predictors of technological quality of meat have not significantly changed in Large White from 1965 to I973. The assumptions underlying the validity of these estimations of genetic change are discussed. 


\section{Références bibliographiques}

BERESKIN B., DAVEY R. J., I976. Breed, line, sex and diet effects and interactions in swine carcass traits. J. Anim. Sci., 42, 43-51.

Chadwick J. P., Smith W. C., LaIRd R., I976. Response to index selection for economy of production and carcass lean content in Large White pigs. Anim. Prod., 22, I64-165 (Abstr.).

COLLEAU J. J., I975a. La mesure de l'évolution génétique des populations au moyen de lignéestémoins. In Bull. tech. Dép. Génét. anim. (Inst. natn. Rech. agron., Fr.) no ${ }^{\circ} 19,4-20$.

ColleaU J. J., 1975 $b$. Méthodes d'estimation des variations génétiques dans le temps sans population témoin. In Bull. tech. Dép. Génét. anim. (Inst. natn. Rech. agron., Fr.) no 19, 26-43.

COOK G. L., SMITH D. H., STEANE D. E., I97I. The progress and penetration of the accreditation scheme in Britain, 1966-1970. Io Congrès international de Zootechnie, 17-23 juillet 1971, Versailles, $7 \mathrm{pp}$.

Cox D. F., SmIYr C., I968. Herd differences and genetic trends in Iowa pigs. J. Anim. Sci., 27, $577-583$.

Czajewska Z. J., Ollivier L., I969. Note sur l'utilisation des résultats des épreuves de descendance par les sélectionneurs de porc en France de 1956 à 1965 . Ann. Génét. Sél. anim., 1, 7 I-77.

DAVEY R. J., BERESKIN B., I978. Genetic and nutritional effects on carcass chemical composition and organ weights of market swine. J. Anim. Sci., 46, 992-rooo.

England M. E., Cunningham P. J., Mandigo R. W., Zimmerman D. R., 1977. Selection for ovulation rate in swine : correlated response in carcass traits. J. Anim. Sci., 45, 983-988.

GARNETr I., RAHNEFEID G. W., 1976. Mass selection for post-weaning growth in swine. V. Correlated response of reproductive traits and pre-weaning growth. Can. J. Anim. Sci., 56, 79I-8or.

Gray R. C., Tribble L. F., Day B. N., Lasley J. F., 1965. Five generations of selection for thinner backfat. J. Anim. Sci., 24, 848 (Abstr.).

HARVEY W. R., I975. Least-squares analysis of data with unequal subclass numbers. ARS H-4, Data systems application division, Agricultural Research Service, U.S. Department of Agriculture, I57 pp.

HETZER H. O., MIL,LER L. R., I973. Selection for high and low fatness in swine: correlated responses of various carcass traits. J.Anim. Sci., 37, I 289-1 30r.

HETZER H. O., MILLER R. H., 1970. Influence of selection for high and low fatness on reproductive performance in swine. J. Anim. Sci., 30, 48I-495.

HILL W. G., I972a. Estimation of genetic change. I. General theory and design of control populations. Anim. Breed. Abstr., 40, I-I 5 .

HILL W. G., I972b. Estimation of realised heritabilities from selection experiments. I. Divergent selection. Biometrics, 28, 747-765.

Houix Y., DaNdo P., SElíler P., r975. Note préliminaire sur l'estimation, à l'aide d'une lignéetémoin, de l'évolution génétique des caractères d'engraissement et de carcasse du porc Lavge White en France, de 1965 à 1973 . In Journées de la Recherche Porcine en France 1975, 279284, Institut technique du Porc, Paris.

JoNES D. W., 1978. Control herds. Meat and Livestock Commission, Milton Keynes, U. K. : report from the Statistics Dept. (2 pp +4 tabl.).

LFGAUL'T C., I971. Corrélations entre les performances d'engraissement et de carcasse et les performances d'élevage chez le Porc. Ann. Génét. Sél. anim., 3, 153-160.

MEAT AND LIVESTOCK COMMISSION, I974. Control herds. Report from the Statistics Department on a comparaison of pigs from the control herds with pigs from the MLC Improvement Scheme (2 $\mathrm{pp}+4$ tabl.).

MOLENAT M., Houix Y., POUl.ENC J., 1974. Contrôles d'engraissement et de carcasse en station chez les porcins : bilan et réflexions (1967-1973). Bull. tech. Dép. Génét. anim. (Inst. natn. Rech. agron., Fr.), n $\mathrm{n}^{0} \mathrm{I} 8$.

MolENat M., NAveaU J., I97o. Bilan zootechnique des stations de contrôle individuel et de contrôle de descendance. In Journées de la Recherche Porcine en France 1970, 227-231, Institut technique du Porc, Paris.

Morris C. A., I975. Genetic relationships of reproduction with growth and with carcass traits in British pigs. Anim. Prod., 20, 31-44. 
Naveau J., I97I. Le progrès génétique dans les troupeaux de sélection de i966 à I970. Bulletin de l'Institut Technique du Porc, I97I (3), I3-I6.

Newton J. R., Cunningham P. J., Zimmerman D. R., 1977. Selection for ovulation rate in swine : correlated response in age and weight at puberty, daily gairi and probe backfat. $J$. Anim. Sci., 44, 30-35.

Olifivier L., I970. L'épreuve de la descendance chez le Porc Large White français de I953 à 1966. I. Analyse de la variation. Ann. Génét. Sél. anim., 2, 31 I-324.

OLIIVIER L., I973. Five generations of selection for increasing litter size in swine. Genetics, 74 (suppl. 2, part 2), 202-203 (Abstr.).

Olirvier L., I974. L'épreuve de la descendance chez le Porc Large White frariçais de I953 à 1966. III. Progrès génétique. Ann. Génét. Sél. anim., 6, 477-492.

OLLIVIER L., I977. Dix ans d'une expérience de sélection individuelle sur des verrats utilisés en insémination artificielle. I. Réponses observées sur des caractères de croissance, de carcasse et de qualité de viande. Ann. Génét. Sél. anim., 9, 353-377.

Olitivier L., LEgault C., Moleinat M., Sellier P., 1978. Les recherches en génétique porcine et leurs applications : un bilan de la période I969-I977. In Io es Journées de la Recherche Porcine en France 1978, 27-42, Institut technique du Porc, Paris.

SELLIER P., I977. Valeur en croisement de verrats Large White et Piétrain; influence du poids d'abattage. In Journées de la Recherche Porcine en France 1977, 85-89, Institut technique du Porc, Paris.

Smirh C., I962. Estimation of genetic change in farm livestock using field records. Anim. Prod., 4, 239-25I.

SMITH C., I963. Genetic change of backfat thickness in the Danish Landrace breed of pigs from 1952 to 1960. Anim. Prod., 5, 259-268.

Sмiтн C., 1976. Alternative forms of genetic controls. Anim. Prod., 23, 403-4I2.

SMITH C., 1977. Use of stored frozen semen and embryos to measure genetic trends in farm livestock. Z. Tievzücht. ZüchtBiol., 94, I I9-127.

VANGEN O., I977. Correlated responses in a selection experiment with pigs after ro years of selection for growth rate and backfat thickness. $28 \mathrm{e}$ Réunion annuelle de la Fédération Européenne de Zootechnie, 22-25 Aout 1977, Bruxelles, I4 pp.

WEBB A. J., KING J. W. B., I976. Development of a synthetic pig sire line by selection with immigration. I. Results of selection and heritability estimates. Anim. Prod., 22, 23 I-244. 